\title{
Exudative Type 3 Retinal Arteriovenous Malformation in a Pediatric Patient
}

\author{
Helena Dens Ingele Casteels \\ Department of Ophthalmology, University Hospitals Leuven, Leuven, Belgium
}

\section{Keywords}

Retinal arteriovenous malformation · Optical coherence tomography angiography · Child

\begin{abstract}
We describe a 7-year-old girl who developed exudation nasally to the right optic disc due to retinal arteriovenous malformation. Fluorescein angiography, spectral domain optical coherence tomography, and optical coherence tomography angiography were performed. We give an overview of the different imaging techniques and discuss the differential diagnosis. Since there was no visual impairment, no treatment was started. A spontaneous decrease in edema and exudation was noted after 6 months.

(C) 2018 The Author(s)

Published by S. Karger AG, Basel
\end{abstract}

\section{Introduction}

Retinal arteriovenous malformation is a primary retinal vascular abnormality. It appears as dilated and tortuous retinal vessels, emerging at the optic disc.

Archer et al. [1] classified arteriovenous malformations into three groups: group 1 with an abnormal capillary plexus between arteries and veins, group 2 with distinctive artery and vein but without capillary plexus, and group 3 without distinction between artery and vein.

New imaging techniques, such as OCT-A, play an important role in the differential diagnosis of other vascular pathologies. We present the first OCT-A performed in a child with retinal arteriovenous malformation. 


\section{Case Presentation}

A 7-year-old white Caucasian girl was referred by her local ophthalmologist to our department for further examination of an abnormal fundoscopic appearance of the right eye.

She did not have any ophthalmologic complaints. Her previous history was unremarkable, except for asthma, which was treated with fluticasone and montelukast. She was wearing glasses for mild hyperopia and astigmatism.

Vision was 20/20 for distance and near in both eyes with correction. Amsler grid showed no metamorphopsia. Biomicroscopy, eye pressure, cover testing, color testing, and pupillary reflexes were normal.

Fundoscopy showed a normal optic disc, macula, and vasculature in the left eye. Papillary edema, dilated and tortuous vessels, without distinctive artery and vein were noticed in the right eye. Based on our findings, the patient can be classified as Archer type 3 arteriovenous malformation. Magnetic resonance imaging excluded associated intracranial pathology (Wyburn-Mason syndrome).

After 4 years of uneventful follow-up, exudation appeared nasally to the optic disc and was confirmed by spectral domain optical coherence tomography (OCT) (Fig. 1a, HRA + OCT SPECTRALIS ${ }^{\circledR}$; Heidelberg Engineering, Heidelberg, Germany). Fluorescein angiography (FA) showed late perivenous staining on the nasal side of the optic disc (Fig. 1b, HRA + OCT SPECTRALIS ${ }^{\circledR}$; Heidelberg Engineering, Heidelberg, Germany). OCT-A revealed dilated and tortuous vessels without a neovascular network (Fig. 2; AngioPlex ${ }^{\circledR}$; ZEISS, Oberkochen, Germany).

Von Hippel-Lindau disease with associated retinal capillary hemangioma was part of our differential diagnosis but could be excluded by genetic screening. Since the exudation was not associated with visual loss, treatment was not initiated. Follow-up 6 months later showed a spontaneous decrease in exudation.

\section{Discussion}

Arteriovenous malformations of the retina should be distinguished from other vascular tumors such as capillary hemangioma, cavernous hemangioma, and vasoproliferative tumor [2]. The characteristic findings of these pathologies on FA and OCT-A will be discussed.

In retinal arteriovenous malformation, the arteriovenous connections are visible on FA, usually without exudation or leakage [2]. In our case, there was perivenous staining on the nasal side of the optic disc. As in the 2 children reported in 2015 [3], our patient showed multiple large inner retinal vessels with prominent shadowing artifact on spectral domain OCT.

This is the first report of OCT-A in a child with retinal arteriovenous malformation. It is the second report of OCT-A in Archer type 3. The other case is a 44-year-old female with a combination of type 3 retinal arteriovenous malformation and leaking retinal arterial macroaneurysm successfully treated with anti-vascular endothelial growth factor [4]. OCT-A in type 2 retinal arteriovenous malformation in the left eye of a 46-year-old male has also been published [5]. In all these patients, dilated tortuous vessels were visible in the more superficial retinal layers on OCT-A.

A round orange-to-red lesion, often accompanied with feeder vessels and exudation, characterizes retinal capillary hemangioma. FA shows early hyperfluorescence and late leakage [2]. OCT-A shows bright tumors in the superficial capillary plexus and sections even more superficial. Few dark spots within the tumor are probably intercapillary areas [6]. 
Cavernous hemangiomas appear as a cluster of dilated vascular channels, usually without exudation. FA shows delayed filling during the venous phase without leakage [2]. OCT-A shows a tumor with multiple nodular/grape-like changes, which are moderately reflective [7].

Retinal vasoproliferative tumors present as a pink-to-yellow raised mass without feeder vessels. Exudation is often present. FA shows early hyperfluorescence and late leakage [2]. OCT-A has not yet been described.

Advantages of OCT-A are the ability to define the depth of the lesion, rapid picture acquisition, high repeatability, high resolution, and easy follow-up of the lesions during treatment, without the risk, although rare, of anaphylaxis. Disadvantages are the smaller field of view and frequent formation of motion artifacts [6, 8]. Our patient showed edema on the nasal side of the right optic disc.

Soliman et al. [9] and Winter et al. [10] proposed that a flow at high velocity and therefore high intraluminal pressure causes leakage and intraretinal edema on the venous side of the communication or adjacent capillaries. They described retinal photocoagulation and anti-vascular endothelial growth factor injections as successful treatment modalities in adults.

Since the fovea was not involved, there was no visual loss in our patient. No treatment was started, and after 6 months, spontaneous decrease in exudation was noted.

\section{Conclusion}

Retinal arteriovenous malformation is a primary retinal vascular abnormality. FA and OCT-A help distinguish arteriovenous malformations from other vascular tumors. Intraretinal edema can be treated with laser therapy or anti-VEGF injections if visual impairment due to macular involvement is present. If there is no visual impairment, watchful waiting is possible, since spontaneous resolution can occur.

\section{Statement of Ethics}

The authors have no ethical conflicts to disclose.

\section{Disclosure Statement}

The authors have no conflicts of interest to declare.

\section{Funding Sources}

The authors have no funding sources to disclose.

\section{Author Contributions}

Helena Dens interpreted all data of the patient and drafted the work. She gave final approval of the version to be published. She is accountable for all aspects of the work. 
Ingele Casteels provided examination and follow-up of the patient. She revised the work critically. She gave final approval of the version to be published. She is accountable for all aspects of the work.

\section{References}

1 Archer DB, Deutman A, Ernest JT, Krill AE. Arteriovenous communications of the retina. Am J Ophthalmol. 1973 Feb;75(2):224-41.

2 Knutsson KA, De Benedetto U, Querques G, Del Turco C, Bandello F, Lattanzio R. Primitive retinal vascular abnormalities: tumors and telangiectasias. Ophthalmologica. 2012;228(2):67-77.

3 Chowaniec MJ, Suh DW, Boldt HC, Stasheff SF, Beer PM, Barry GP. Anomalous optical coherence tomography findings in Wyburn-Mason syndrome and isolated retinal arteriovenous malformation. J AAPOS. 2015 Apr;19(2):175-7.

4 Vishal R, Avadesh O, Srinivas R, Taraprasad D. Retinal racemose hemangioma with retinal artery macroaneurysm: optical coherence tomography angiography (OCTA) findings. Am J Ophthalmol Case Rep. 2018 Jun;11:98-100.

5 Sharma P, Sridhar J, Rayess N, Maguire JI. Optical coherence tomography angiography (OCT-A) of type 2 retinal arteriovenous malformation. Can J Ophthalmol. 2015 Oct;50(5):e93-6.

6 Sagar P, Rajesh R, Shanmugam M, Konana VK, Mishra D. Comparison of optical coherence tomography angiography and fundus fluorescein angiography features of retinal capillary hemangioblastoma. Indian J Ophthalmol. 2018 Jun;66(6):872-6.

7 Lyu S, Zhang M, Wang RK, Gao Y, Zhang Q, Min X. Analysis of the characteristics of optical coherence tomography angiography for retinal cavernous hemangioma: A case report. Medicine (Baltimore). 2018 Feb;97(7):e9940.

8 Wylęgała A, Teper S, Dobrowolski D, Wylęgała E. Optical coherence angiography: A review. Medicine (Baltimore). 2016 Oct;95(41):e4907.

9 Soliman W, Haamann P, Larsen M. Exudation, response to photocoagulation and spontaneous remission in a case of bilateral racemose haemangioma. Acta Ophthalmol Scand. 2006 Jun;84(3):429-31.

10 Winter E, Elsås T, Austeng D. Anti-VEGF treating macular oedema caused by retinal arteriovenous malformation - a case report. Acta Ophthalmol. 2014 Mar;92(2):192-3. 


\section{Case Reports in Ophthalmology}
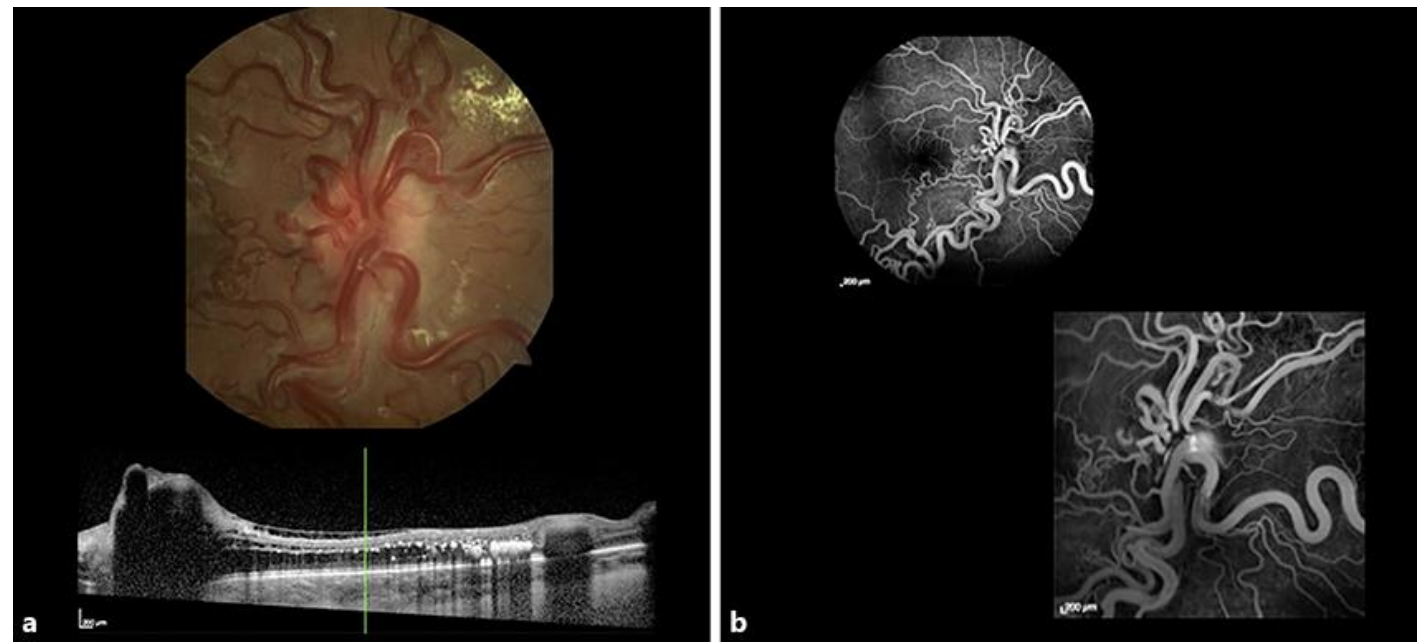

Fig. 1. a Fundus photograph showing exudation nasally to the optic disc of the right eye, and OCT (HRA + OCT SPECTRALIS ${ }^{\circledR}$, Heidelberg Engineering, Heidelberg, Germany) showing exudation and edema on the nasal side of the optic disc. b FA (HRA + OCT SPECTRALIS ${ }^{\circledR}$, Heidelberg Engineering, Heidelberg, Germany) showing perivenous staining on the nasal side of the optic disc. 


\section{Case Reports in Ophthalmology}

Case Rep Ophthalmol 2018;9:504-509

DOI: $10.1159 / 000495656$

(c) 2018 The Author(s). Published by S. Karger AG, Basel www.karger.com/cop

Dens and Casteels: Exudative Type 3 Retinal Arteriovenous Malformation in a Pediatric Patient

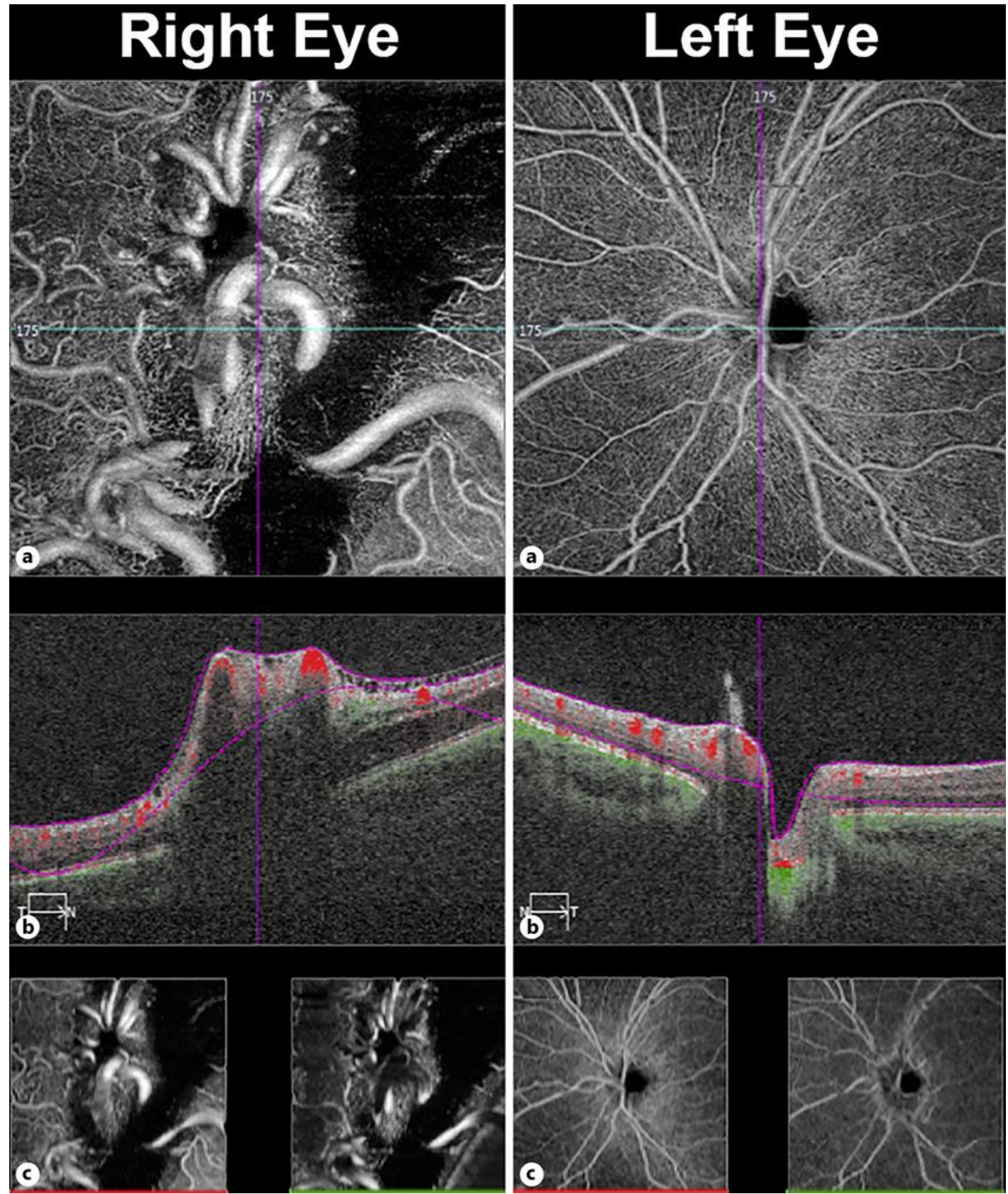

Fig. 2. a OCT-A (AngioPlex ${ }^{\circledR}$, ZEISS, Oberkochen, Germany) showing dilated and tortuous vessels in the right eye without a neovascular network, compared to the normal vessels of the left eye. $\mathbf{b}$ Corresponding profiles and vascular flow patterns. c OCT-A showing superficial retinal layers (bottom left, red line) and deep retinal layers (bottom right, green line) of the right and left eye, respectively. 